\title{
Cemented Bipolar Hemiarthroplasty Provides Definitive Treatment for Femoral Neck Fractures at 20 Years and Beyond
}

\author{
Philipp von Roth MD, Matthew P. Abdel MD, W. Scott Harmsen MS, \\ Daniel J. Berry MD
}

Received: 18 March 2015/Accepted: 9 July 2015/Published online: 18 July 2015

(C) The Association of Bone and Joint Surgeons (B) 2015

\begin{abstract}
Background Displaced femoral neck fractures frequently are treated with bipolar hemiarthroplasties. Despite the frequency with which bipolar hemiarthroplasty is used to treat these fractures, there are few long-term data.

Questions/purposes We sought to evaluate (1) the cumulative incidence of revision for any reason of bipolar hemiarthroplasties at 20 years, and the proportion of patients who lived more than 20 years who still have the prosthesis in situ from the index arthroplasty, (2) the cumulative incidence of aseptic loosening at 20 years, and (3) the Harris hip score of the surviving patients at long term.
\end{abstract}

One of the authors certifies that he (DJB), or a member of his or her immediate family, has or may receive payments or benefits, during the study period, an amount of USD 100,001-USD 1,000,000 from Depuy Orthopaedics, Warsaw, IN, USA).

All ICMJE Conflict of Interest Forms for authors and Clinical Orthopaedics and Related Research ${ }^{\circledR}$ editors and board members are on file with the publication and can be viewed on request.

Clinical Orthopaedics and Related Research ${ }^{\mathbb{R}}$ neither advocates nor endorses the use of any treatment, drug, or device. Readers are encouraged to always seek additional information, including FDAapproval status, of any drug or device prior to clinical use.

Each author certifies that his or her institution approved the human protocol for this investigation, that all investigations were conducted in conformity with ethical principles of research, and that informed consent for participation in the study was obtained.

This work was performed at the Mayo Clinic, Rochester, MN, USA.

P. von Roth

Department of Orthopedic Surgery, Charité Universitätsmedizin

Berlin, Berlin, Germany

M. P. Abdel ( $\square)$, W. S. Harmsen, D. J. Berry

Department of Orthopedic Surgery, Mayo Clinic, 200 First Street

SW, Rochester, MN 55905, USA

e-mail: abdel.matthew@mayo.edu
Methods We performed 376 cemented bipolar hemiarthroplasties for displaced femoral neck fractures in 359 patients between 1976 and 1985. At a minimum of followup of 20 years (mean, 24 years; range, 20-31 years), 339 of 359 patients (94\%) were deceased, leaving 20 patients in the study group. Of those, one patient was confirmed to be lost to followup and two others had radiographic followup only. Three hundred fifty-nine patients (99.2\%) (376 of the original 379 hips) were followed until death, revision of the hemiarthroplasty, or for at least 20 years (of clinical followup). Bipolar hemiarthroplasty was performed for displaced femoral neck fractures. Cemented fixation was the standard of care between 1976 and 1985 at our institution. The mean age of the patients at the time of surgery was 79 years (range, 6099 years). The cumulative incidence of revision, estimated with death as a competing risk, and radiographs were evaluated for signs of aseptic loosening by a surgeon not involved in the clinical care of the patients. Clinical function was evaluated with the Harris hip score. The mean age of the patients at the time of surgery was 79 years.

Results The 20-year cumulative incidence of revision for any reason was $3.5 \%$ (95\% CI, $1.6 \%-5.3 \%$ ). Of the 20 patients who survived more than 20 years, seven had the implant intact. The 20-year cumulative incidence of revision for aseptic loosening was $1.4 \%$ (95\% CI, $0.2 \%-2.6 \%$ ). The mean Harris hip score in patients who were still living and patient who did not have revision surgery was $63 \pm 22$, however 13 of the 20 patients had undergone revision surgery.

Conclusions The long-term survivorship of bipolar hemiarthroplasty prostheses used to treat displaced femoral neck fractures in the elderly was high, and the procedure can be considered definitive for the majority of elderly patients with a femoral neck fracture. In this series, $6 \%$ 
(20 patients, 339 of 359) of the patients survived more than 20 years after treatment of a femoral neck fracture with a bipolar hemiarthroplasty. Of those, 35\% (seven of 20) survived with their index prosthesis in situ.

Level of Evidence Level IV, therapeutic study.

\section{Introduction}

Displaced femoral neck fractures are common injuries, which are increasing in prevalence [2]. In elderly patients, the fractures often are treated with unipolar or bipolar hemiarthroplasty or THA [5, 7, 12, 16, 24]. Studies have shown satisfactory short- to mid-term results of bipolar hemiarthroplasties in terms of survival and generally highvalidated outcomes scores in elderly patients with femoral neck fractures [5, 7, 12]. However, there are few studies regarding long-term implant performance and survivorship in this patient population [22, 25].

Controversy exists regarding the relative merits of hemiarthroplasty versus THA for patients with displaced femoral neck fractures, with some data supporting improved function in selected patients treated with THA [1, $3,4,6,8,10,13,17,18,26]$. It is important to understand short- and mid-term clinical results and the long-term likelihood of reoperation or revision of two commonly used treatment options, THA and bipolar hemiarthroplasty $[12,18,26]$.

The purpose of the current study was to evaluate (1) the cumulative incidence of revision for any reason of bipolar hemiarthroplasty at 20 years, and the proportion of patients who lived more than 20 years who still have the index prosthesis in situ, (2) the cumulative incidence of aseptic loosening at 20 years, and (3) the Harris hip score of the surviving patients at long term.

\section{Patients and Methods}

The study group included all patients who had a cemented bipolar hemiarthroplasty, at one institution, for an acute displaced intracapsular femoral neck fracture. The study included 376 cemented bipolar hemiarthroplasties for displaced femoral neck fractures in 359 patients between 1976 and 1985. At a minimum followup of 20 years (mean, 24 years; range, 20-31 years), 339 of 359 patients (94\%) had died, leaving 20 patients in the study group. Of those, one patient was confirmed to be lost to followup, and two others had radiographic followup only. The mean age of patients who were living and who did not have revision surgery was 91 years (range, 88-96 years). Three hundred fifty-nine patients (99.2\%) (376 of the original 379 hips) were followed until revision surgery or for at least 20 years (of clinical followup). The mean followup for patients who died was 6 years (range, $0-23$ years). The mean age of the patients at the time of surgery was 79 years (range, 60-99 years). Patient information was collected prospectively for all patients and entered in an institutional total joint registry. Institutional review board approval was obtained before initiation of the study.

The operative approach was anterolateral in $286(76 \%)$, posterior in $73(19 \%)$, and transtrochanteric in $17(5 \%)$ procedures. The following stems were used: Bateman stem (3M, St Paul, MN, USA) in 214 cases; Omnifit stem in 74 cases (formerly Osteonics, now Stryker, Mahwah, NJ, USA); Harris Design-2 stem (formerly Howmedica, now Stryker) in 62 cases; Harris Precoat stem (Zimmer, Warsaw, IN, USA) in 19 hips; Austin-Moore straight stem $(3 \mathrm{M})$ in four hips, and 60/32 stem (Zimmer) in three hips.

Bipolar hemiarthroplasty was performed for displaced femoral neck fractures. During the study period no unipolar hemiarthroplasties were performed. Exclusion criteria were pathologic fractures, nonunions, or prior failed open reduction and internal fixation. Cemented fixation was the standard of care between 1976 and 1985 at our institution.

The following bipolar component designs were used: Bateman component (3M) in 276 cases, UHR component (formerly Osteonics, now Stryker) in 76 cases, Bi-Articular component (Zimmer) in 14 cases, and Bi-Centric component (formerly Howmedica, now Stryker) in 10 cases.

Clinical followup was performed routinely at 1,2 , and 5 years, and every 5 years thereafter, and patients were assessed using the Harris hip score. When patients could not be evaluated in person, a questionnaire was used and patients were asked to send radiographs.

Acetabular protrusio and cartilage erosion were evaluated as described by LaBelle et al. [15]. In brief, acetabular cartilage erosion was assessed by measuring the change of thickness of the acetabular cartilage compared with the postoperative radiograph [15]. Femoral component loosening was assessed on radiographs by measuring radiolucencies at the cement-bone or prosthesis-cement interface following the methods of Gruen et al. [9].

\section{Statistical Methods}

The cumulative incidence was estimated using a competing risk analysis where death was considered the competing risk for free of revision for wear or mechanical failure, free of revision for aseptic loosening, free of any revision, and free of reoperation. The association of patient factors with the risk of revision was assessed using Cox proportional hazards regression. The alpha level was set at 0.05 for statistical significance. 


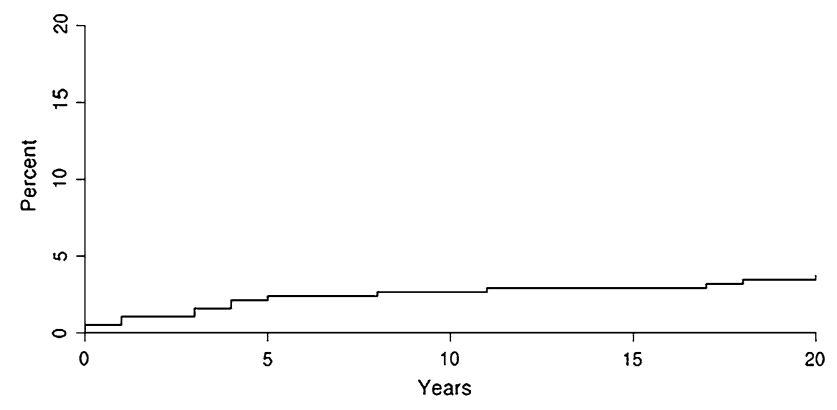

Fig. 1 The graph shows the cumulative incidence of revision when patient death was considered as a competing risk (20-year rate $=$ $3.5 \%)$.

Table 1. Summary of the 20-year cumulative incidence rates

\begin{tabular}{lll}
\hline Reason for revision & $\begin{array}{l}\text { 20-year survival } \\
\text { rate }(\%)\end{array}$ \\
\hline Articular cartilage wear & 1.4 & $0.2 \%-2.5 \%$ \\
Aseptic femoral component loosening & 1.4 & $0.2 \%-2.6 \%$ \\
Any revision & 3.5 & $1.6 \%-5.3 \%$ \\
Any reoperation & 4.3 & $2.2 \%-6.9 \%$ \\
\hline
\end{tabular}

\section{Results}

The 20-year cumulative incidence of revision for any reason was 3.5\% (95\% CI, 1.6\%-5.3\%) (Fig. 1), and the 20year cumulative incidence of any reoperation was $4.3 \%$ (95\% CI, 2.2\%-6.9\%). Seven of the 20 patients who survived more than 20 years had the implant intact.

The 20-year cumulative incidence of revision for aseptic femoral component loosening was $1.4 \%$ (95\% CI, $0.2 \%-$ $2.6 \%)$. The 20-year cumulative incidence of revision for articular cartilage wear was $1.4 \%(95 \% \mathrm{CI}, 0.2 \%-2.5 \%)$ (Table 1). Age at operation $(\mathrm{p}=0.22)$, sex $(\mathrm{p}=0.14)$, and operative approach $(p=0.64)$ were not predictors of implant survivorship. Of patients who were alive and those without revision surgery at last followup, two had less than 1-mm acetabular cartilage erosion. Otherwise, no living patients showed evidence of cartilage wear, and none of the femoral components had evidence of loosening.

The mean Harris hip score in patients who were living and those without revision surgery was $63 \pm 22$; however, as mentioned previously, 13 of the 20 patients had undergone revision surgery.

\section{Discussion}

Femoral neck fractures are among the leading causes of mortality and disability and their prevalence is expected to increase [2]. Treatment options include THA and bipolar hemiarthroplasty $[2,12,18,26]$. The optimal surgical treatment of displaced femoral neck fractures is still debated. There is lack of knowledge regarding the longterm implant performance and survivorship in elderly patients with displaced femoral neck fractures treated with bipolar hemiarthroplasty.

Therefore, the purpose of our study was to evaluate the long-term durability of bipolar hemiarthroplasties in elderly patients. The 20-year cumulative incidence of revision for any reason was 3.5\% using a competing risk analysis, where death was considered the competing risk. The procedure can be considered definitive for the life of the majority of elderly patients with a femoral neck fracture.

Our study has some limitations. First, we included only cemented femoral components. During the period of index surgery between 1976 and 1985, the cemented bipolar hemiarthroplasty was the standard of care for displaced femoral neck fractures for the elderly and patient with low demands. No unipolar components were used during the study period for the indication of a displaced femoral neck fracture. Developments in implant fixation techniques have led to the use of uncemented stems in hemiarthroplasties, although studies have shown advantages in the survival with cemented femoral components for this elderly cohort $[6,18,26,27]$. Second, our intention was not to compare cemented bipolar hemiarthroplasties with THAs. Although we report Harris hip scores, we did not collect more indepth patient-reported outcomes scores. As this is a final followup report at 20 years after the index surgery, the patient cohort consisted of few patients who did not have revision surgery and few living patients; however, to our knowledge, this study has the largest patient cohort with the longest followup for this commonly performed procedure for this common diagnosis.

To our best knowledge, this study is the first regarding a competing risk model of cemented bipolar hemiarthroplasty at 20 years. The results presented can serve as a benchmark for comparison to components with cementless fixation, which are more commonly used today. In this study, we present the long-term implant survivorship of 376 bipolar hemiarthroplasties, all performed for displaced intracapsular femoral neck fractures, in contrast to studies that report short- or mid-term results. At 20 years, the cumulative incidence of revision for any reason was only $3.5 \%$, where death was considered a competing risk. This inherently indicates that deep periprosthetic infection was not a major concern in this elderly cohort. This may be related to the use of antibiotic-loaded bone cement. Of the patients who survived more than 20 years after the index surgery, 35\% had an unrevised component in situ. Comparable studies do not report on the outcome for patients who are living and those who have not undergone revision surgery $[6,11,15,20]$. LaBelle et al. [13] reported a $10 \%$ 
implant failure rate at 7 years in 128 cemented bipolar hemiarthroplasties. An analysis of the Hospital Episode Statistics database of England found an implant survival rate of $98 \%$ after 4 years in 3866 patients undergoing hemiarthroplasty [1]. Gjertsen et al. [6] found survival rates of $97 \%$ at 5 years in 8639 patients with cemented bipolar hemiarthroplasties from the Norwegian Arthroplasty Register.

Complementing our survivorship investigation, a detailed radiologic analysis was performed. The radiographic data showed that the implants continued to function well mechanically at long-term followup in the few remaining patients. Because some studies of bipolar hemiarthroplasties are registry-based, they do not include a radiographic analysis [6, 12, 13, 19].

The Harris hip score was 63 in patients who are alive and who have not had revision surgery $(n=7)$. Harris hip scores were not reported in numerous studies [6, 11, 15, 20, 27] after bipolar hemiarthroplasty. However, Ritter et al. [23] showed an age-related decline of the Harris hip score for THA of 0.88 points per year even when patients otherwise were doing well. Keeping in mind the mean age of living patients and those without revision surgery (91 years), the relatively low Harris hip score can be explained by the age-related disability of the individuals. Studies comparing two different types of surgical treatment (eg, bipolar vs unipolar hemiarthroplasty) found reasonable results regarding recovery of ambulatory status and activities of daily living for patients with bipolar hemiarthroplasties [14, 21].

At 20 years followup, we found the survivorship of bipolar hemiarthroplasties for displaced femoral neck fractures were good. Patients living more than 20 years after the index surgery have a high risk of undergoing revision surgery. However, the cemented bipolar hemiarthroplasty is a definitive treatment option for the elderly patient in the majority of cases.

\section{References}

1. Bell KR, Clement ND, Jenkins PJ, Keating JF. A comparison of the use of uncemented hydroxyapatite-coated bipolar and cemented femoral stems in the treatment of femoral neck fractures: a case-control study. Bone Joint J. 2014;96:299-305.

2. Bhandari M, Devereaux PJ, Einhorn TA, Thabane L, Schemitsch EH, Koval KJ, Frihagen F, Poolman RW, Tetsworth K, GuerraFarfan E, Madden K, Sprague S, Guyatt G; HEALTH Investigators. Hip fracture evaluation with alternatives of total hip arthroplasty versus hemiarthroplasty (HEALTH): protocol for a multicentre randomised trial. BMJ Open. 2015;5:e06263.

3. Blomfeldt R, Tornkvist H, Eriksson K, Soderqvist A, Ponzer S, Tidermark J. A randomised controlled trial comparing bipolar hemiarthroplasty with total hip replacement for displaced intracapsular fractures of the femoral neck in elderly patients. $J$ Bone Joint Surg Br. 2007;89:160-165.
4. Deangelis JP, Ademi A, Staff I, Lewis CG. Cemented versus uncemented hemiarthroplasty for displaced femoral neck fractures: a prospective randomized trial with early follow-up. J Orthop Trauma. 2012;26:135-140.

5. Frihagen F, Nordsletten L, Madsen JE. Hemiarthroplasty or internal fixation for intracapsular displaced femoral neck fractures: randomised controlled trial. BMJ. 2007;335:1251-1254.

6. Gjertsen JE, Lie SA, Vinje T, Engesaeter LB, Hallan G, Matre K, Furnes O. More re-operations after uncemented than cemented hemiarthroplasty used in the treatment of displaced fractures of the femoral neck: an observational study of 11,116 hemiarthroplasties from a national register. $J$ Bone Joint Surg Br. 2012;94:1113-1119.

7. Gjertsen JE, Vinje T, Engesaeter LB, Lie SA, Havelin LI, Furnes $\mathrm{O}$, Fevang JM. Internal screw fixation compared with bipolar hemiarthroplasty for treatment of displaced femoral neck fractures in elderly patients. J Bone Joint Surg Am. 2010;92:619-628.

8. Goh SK, Samuel M, Su DH, Chan ES, Yeo SJ. Meta-analysis comparing total hip arthroplasty with hemiarthroplasty in the treatment of displaced neck of femur fracture. J Arthroplasty. 2009;24:400-406.

9. Gruen TA, McNeice GM, Amstutz HC. "Modes of failure" of cemented stem-type femoral components: a radiographic analysis of loosening. Clin Orthop Relat Res. 1979;141:17-27.

10. Hedbeck CJ, Enocson A, Lapidus G, Blomfeldt R, Tornkvist H, Ponzer S, Tidermark J. Comparison of bipolar hemiarthroplasty with total hip arthroplasty for displaced femoral neck fractures: a concise four-year follow-up of a randomized trial. J Bone Joint Surg Am. 2011;93:445-450.

11. Isotalo K, Rantanen J, Aarimaa V, Gullichsen E. The long-term results of Lubinus interplanta hemiarthroplasty in 228 acute femoral neck fractures: a retrospective six-year follow-up. Scand J Surg. 2002;91:357-360.

12. Jameson SS, Lees D, James P, Johnson A, Nachtsheim C, McVie JL, Rangan A, Muller SD, Reed MR. Cemented hemiarthroplasty or hip replacement for intracapsular neck of femur fracture? A comparison of 7732 matched patients using national data. Injury. 2013;44:1940-1944.

13. Kannan A, Kancherla R, McMahon S, Hawdon G, Soral A, Malhotra R. Arthroplasty options in femoral-neck fracture: answers from the national registries. Int Orthop. 2012;36:1-8.

14. Kanto K, Sihvonen R, Eskelinen A, Laitinen M. Uni- and bipolar hemiarthroplasty with a modern cemented femoral component provides elderly patients with displaced femoral neck fractures with equal functional outcome and survivorship at medium-term follow-up. Arch Orthop Trauma Surg. 2014;134:1251-1259.

15. LaBelle LW, Colwill JC, Swanson AB. Bateman bipolar hip arthroplasty for femoral neck fractures: a five- to ten-year followup study. Clin Orthop Relat Res. 1990;251:20-25.

16. Leonardsson O, Sernbo I, Carlsson A, Akesson K, Rogmark C. Long-term follow-up of replacement compared with internal fixation for displaced femoral neck fractures: results at ten years in a randomised study of 450 patients. J Bone Joint Surg Br. 2010;92:406-412.

17. Li T, Zhuang Q, Weng X, Zhou L, Bian Y. Cemented versus uncemented hemiarthroplasty for femoral neck fractures in elderly patients: a meta-analysis. PLoS One. 2013;8:e68903.

18. Luo X, He S, Li Z, Huang D. Systematic review of cemented versus uncemented hemiarthroplasty for displaced femoral neck fractures in older patients. Arch Orthop Trauma Surg. 2012;132:455-463.

19. Miller BJ, Lu X, Cram P. The trends in treatment of femoral neck fractures in the Medicare population from 1991 to 2008. J Bone Joint Surg Am. 2013;95:e132.

20. Miller D, Choksey A, Jones P, Perkins R. Medium to long term results of the Exeter bipolar hemiarthroplasty for femoral neck 
fractures in active, independent patients: 5-13 year follow-up. Hip Int. 2008;18:301-306.

21. Ong BC, Maurer SG, Aharonoff GB, Zuckerman JD, Koval KJ. Unipolar versus bipolar hemiarthroplasty: functional outcome after femoral neck fracture at a minimum of thirty-six months of follow-up. J Orthop Trauma. 2002;16:317-322.

22. Parker MI, Pryor G, Gurusamy K. Cemented versus uncemented hemiarthroplasty for intracapsular hip fractures: a randomised controlled trial in 400 patients. J Bone Joint Surg Br. 2010;92:116-122.

23. Ritter MA, Thong AE, Davis KE, Berend ME, Meding JB, Faris PM. Long-term deterioration of joint evaluation scores. $J$ Bone Joint Surg Br. 2004;86:438-442.

24. Rogmark C, Carlsson A, Johnell O, Sernbo I. A prospective randomised trial of internal fixation versus arthroplasty for displaced fractures of the neck of the femur: functional outcome for 450 patients at two years. J Bone Joint Surg Br. 2002;84:183188.

25. Sonne-Holm S, Walter S, Jensen JS. Moore hemi-arthroplasty with and without bone cement in femoral neck fractures: aclinical controlled trial. Acta Orthop Scand. 1982;53:953-956.

26. Taylor F, Wright M, Zhu M. Hemiarthroplasty of the hip with and without cement: a randomized clinical trial. J Bone Joint Surg Am. 2012;94:577-583.

27. Viberg B, Overgaard S, Lauritsen J, Ovesen O. Lower reoperation rate for cemented hemiarthroplasty than for uncemented hemiarthroplasty and internal fixation following femoral neck fracture: 12 - to 19 -year follow-up of patients aged 75 years or more. Acta Orthop. 2013;84:254-259. 